Bull. Austral. Math. Soc.

Vol. 40 (1989) [59-77]

\title{
IDENTITIES FOR EXISTENCE VARIETIES OF REGULAR SEMIGROUPS
}

\author{
T.E. HaLl
}

\begin{abstract}
A natural concept of variety for regular semigroups is introduced: an existence variety (or e-variety) of regular semigroups is a class of regular semigroups closed under the operations $H, S_{e}, P$ of taking all homomorphic images, regular subsemigroups and direct products respectively. Examples include the class of orthodox semigroups, the class of (regular) locally inverse semigroups and the class of regular $E$-solid semigroups. The lattice of $e$-varieties of regular semigroups includes the lattices of varieties of inverse semigroups and of completely regular semigroups. A Birkhoff-type theorem is proved, showing that each $e$-variety is determined by a set of identities: such identities are then given for many $e$-varieties. The concept is meaningful in universal algebra, and as for regular semigroups could give interesting results for $e$-varieties of regular rings.
\end{abstract}

\section{Preliminaries}

A semigroup $S$ (or $(S, \cdot)$, though we will always replace $\cdot$ by juxtaposition) is called regular (in the sense of von Neumann for rings) if for each $a \in S$ there exists $x \in S$ such that $a x a=a$. The element $y=x a x$ then satisfies both aya $=a$ and $y a y=y$, and is called an inverse of $a$ in $S$. A semigroup is called an inverse semigroup if each element has a unique inverse, and a semigroup $S$ (not necessarily regular) is called a locally inverse semigroup if for each idempotent $e \in S$, the subsemigroup eSe is inverse.

We follow the notation and conventions of Clifford and Preston [2] and Howie [9], especially for Green's relations $\mathcal{L}, \mathcal{R}, \mathcal{D}, \mathcal{H}$ and $\mathcal{J}$.

An orthodox semigroup is a regular semigroup in which the idempotents form a subsemigroup. A semigroup $S$ is called $E$-solid if for all idempotents $e, f, g \in S$ such that $e \mathcal{L} f \mathcal{R} g$ there exists an idempotent $h \in S$ such that $e \mathcal{R} h \mathcal{L} g$. Such semigroups include inverse semigroups (in fact, orthodox semigroups) and completely regular semigroups (defined as regular semigroups which are unions of groups).

For any class $\mathcal{C}$ of regular semigroups we define classes $H(\mathcal{C}), S_{e}(\mathcal{C})$ and $P(\mathcal{C})$ as follows: $H(\mathcal{C})$ is the class of all (regular) semigroups that are homomorphic images of

\section{Received 19 September 1988}

Many years ago, the author heard of the idea of considering varieties of regular unary semigroups from B.H. Neumann, via G.B. Preston, and he thanks them both for sharing the idea. The author also thanks L. Polák, G.B. Preston and P.G. Trotter for very helpful suggestions about the exposition of this paper.

Copyright Clearance Centre, Inc. Serial-fee code: $0004-9729 / 89 \$ \$ 2.00+0.00$. 
semigroups in $\mathcal{C} ; \mathbf{S}_{e}(\mathcal{C})$ is the class of all regular subsemigroups of semigroups in $\mathcal{C}$; and $P(\mathcal{C})$ is the class of all direct products of semigroups in $\mathcal{C}$. A class $\mathcal{V}$ of regular senugroups is called an existence variety, or e-variety, if $H(\mathcal{V}) \subseteq \mathcal{V}, \mathrm{s}_{e}(\mathcal{V}) \subseteq \mathcal{V}$ and $\mathbf{P}(\mathcal{V}) \subseteq \mathcal{V}$

\section{A Birkhoff Theorem}

Simultaneously, and quite independently of the present paper, Kadourek and Szendrei [10, Section 1] have considered e-varieties of just orthodox semigroups, which they call bivarieties of orthodox semigroups, and have also obtained a Birkhoff-type theorem. By restricting in a natural way the type of identities considered, they obtain the result that bivarieties of orthodox semigroups are precisely equational classes of orthodox semigroups (their definition of "an orthodox semigroup $(S, \cdot)$ satisfies an identity" is prima facie slightly different, and might not be equivalent: for example, for the word $x x^{\prime} y^{\prime} y$ they can substitute $a a^{\prime} a^{*} a$ with $a^{\prime}, a^{*}$ being different inverses of $a$ ).

For any regular semigroup $(S, \cdot)$ (or more simply $S$ ), there is (by the Axiom of Choice) a unary operation ': $S \rightarrow S$ on $S$ such that, for all $x \in S, x x^{\prime} x=x$ and $x^{\prime} x x^{\prime}=x^{\prime}$; we call such an operation an inverse unary operation. By a unary semigroup we mean an algebra $\left(S, \cdot,{ }^{\prime}\right)$ such that $(S, \cdot)$ is a semigroup and ' is a unary operation on $S$, while by a regular unary semigroup we mean a unary semigroup $(S, \cdot, ')$ such that $(S, \cdot)$ is a regular semigroup and ' is an inverse unary operation on $(S, \cdot)$. We denote the variety of all regular unary semigroups by $\mathcal{R} U \mathcal{S}$, and the e-variety of all regular semigroups by $\mathcal{R S}$. For each class $\mathcal{C}$ of regular semigroups, define a class $\mathcal{C}^{\prime}$ of regular unary semigroups by $\mathcal{C}^{\prime}=\left\{\left(S, \cdot,^{\prime}\right) \in \mathcal{R U S}:(S, \cdot) \in \mathcal{C}\right\}$.

For each element $x$ in a semigroup $S$, we put

$$
V(x)=\{y \in S: x y x=x \& y x y=y\}
$$

the set of inverses of $x$ in $S$. Then the number of inverse unary operations on a regular semigroup $S$ is of course $\prod_{x \in S}|V(x)|$, the product of the cardinalities of the sets $V(x), x \in S$. Thus for each $S$ in $\mathcal{C}$, the $\prod_{x \in S}|V(x)|$ regular unary semigroups obtained from $S$, by adding an inverse unary operation in all possible ways, all belong to $\mathcal{C}^{\prime}$.

THEOREM 2.1. For each existence variety $\mathcal{V}$ of regular semigroups, the class $\mathcal{V}^{\prime}$ is a variety of (regular) unary semigroups.

Proof: Take any $\left(S, \cdot,^{\prime}\right) \in \mathcal{V}^{\prime}$, and any unary semigroup $\left(T, \cdot,^{\prime}\right)$ such that there exists a (unary semigroup) morphism $\phi$ of $\left(S, \cdot,^{\prime}\right)$ onto $\left(T, \cdot,^{\prime}\right)$. Then $\phi$ is also a (semigroup) morphism of $(S, \cdot)$ onto $(T, \cdot)$, so $(T, \cdot) \in \mathcal{V}$, whence $\left(T, \cdot,^{\prime}\right) \in \mathcal{V}^{\prime}$, so $\mathcal{V}^{\prime}$ is closed under $H$, the taking of all homomorplic images. 
Take any $\left(S, \cdot,^{\prime}\right) \in \mathcal{V}^{\prime}$ and any (regular) unary subsemigroup $\left(T, \cdot,^{\prime}\right)$ of $\left(S, \cdot,{ }^{\prime}\right)$. Then $(T, \cdot) \in \mathcal{V}$ so again $\left(T, \cdot,^{\prime}\right) \in \mathcal{V}^{\prime}$, whence $\mathcal{V}^{\prime}$ is closed under $\mathbf{S}$, the taking of all (regular) unary subsemigroups. Entirely similarly, we see that $\mathcal{V}^{\prime}$ is closed under taking all direct products, which completes the proof.

For each e-variety $\mathcal{V}$ of regular semigroups, we denote by $\operatorname{Id}\left(\mathcal{V}^{\prime}\right)$ the set of all (unary semigroup) identities satisfied by all members of $\mathcal{V}^{\prime}$. We now obtain a Birkhoff theorem for e-varieties (in the proof, the brackets indicate an alternative statement).

TheOREM 2.2. For any existence variety $\mathcal{V}$ of regular semigroups, and for any set of (unary semigroup) identities $B$ containing $x x^{\prime} x=x$ and $x^{\prime} x x^{\prime}=x^{\prime}$, the following two conditions are equivalent:

(i) $B$ is a basis of $\operatorname{Id}\left(\mathcal{V}^{\prime}\right)$;

(ii) $\mathcal{V}$ is given by

$$
\begin{gathered}
\mathcal{V}=\{(S, \cdot) \in \mathcal{R S}: \text { for some inverse unary operation 'on } \\
\left.(S, \cdot),\left(S, \cdot,^{\prime}\right) \text { satisfies } B\right\}
\end{gathered}
$$

and by

$$
\begin{gathered}
\mathcal{V}=\{(S, \cdot) \in \mathcal{R} S: \text { for every inverse unary operation ' on } \\
\left.(S, \cdot),\left(S, \cdot,^{\prime}\right) \text { satisfies } B\right\} .
\end{gathered}
$$

Proof: (i) $\Rightarrow$ (ii). Assume (i). If for some [for every] inverse unary operation ' on $(S, \cdot)$, we have that $\left(S, \cdot,^{\prime}\right)$ satisfies $B$, then $\left(S, \cdot,^{\prime}\right)$ is in $\mathcal{V}^{\prime}$ by Birkhoff's Theorem for the variety $\mathcal{V}^{\prime}$, whence $(S, \cdot)$ is in $\mathcal{V}$ by the definition of $\mathcal{V}^{\prime}$. Conversely, if $(S, \cdot)$ is in $\mathcal{V}$ then $\left.(S, \cdot,)^{\prime}\right)$ is in $\mathcal{V}^{\prime}$ (that is, satisfies $B$ ), for every inverse unary operation ' on $(S, \cdot)$, which proves (ii).

(ii) $\Rightarrow$ (i). Assume (ii) and put

$$
\langle B\rangle_{v}=\left\{\left(S, \cdot,^{\prime}\right) \in \mathcal{R U S}:\left(S, \cdot,^{\prime}\right) \text { satisfies } B\right\}
$$

the $\mathcal{R U S}$ variety determined by $B$. From the first equation in condition (ii) we have $\langle B\rangle_{v} \subseteq \mathcal{V}^{\prime}$, and from the second equation in condition (ii) we have that $\mathcal{V}^{\prime} \subseteq\langle B\rangle_{\text {" }}$. Thus $B$ is a basis of $\mathcal{V}^{\prime}=\langle B\rangle_{v}$, as required.

We say that an e-variety $\mathcal{V}$ is strongly determined by a set of identities $B$ if $B$ is a basis of Id $\left(\mathcal{V}^{\prime}\right)$. We say that a regular semigroup $(S, \cdot)$ satisfies a set $C^{\prime}$ of $\mathcal{R U} S$ identities if $\left.(S, \cdot)^{\prime}\right)$ satisfies $C$ for every inverse unary operation' on $(S, \cdot)$. Note that from Theorem 2.2, a basis $B$ of $\operatorname{Id}\left(\mathcal{V}^{\prime}\right)$, for $\mathcal{V}$ an $e$-variety of regular semigroups, has the property that, for each regular semigroup $(S, \cdot),\left(S, \cdot,^{\prime}\right)$ satisfies $B$ for some 
inverse unary operation ' on $(S, \cdot)$ if and only if $\left(S, \cdot,^{\prime}\right)$ satisfies $B$ for every inverse unary operation ' on $(S, \cdot)$.

For each set $C$ of $R \mathcal{R}$ identities, we call the class

$$
\mathcal{E}(C)=\{(S, \cdot) \in \mathcal{R S}:(S, \cdot) \text { satisfies } C\}
$$

an equational class. By Theorem 2.2, each existence variety is an equational class. Example 2.4 below shows that not every equational class is an existence variety. We say that an $e$-variety $\mathcal{V}$ is weakly determined by a set of identities $C$ if $\mathcal{E}(C)=\mathcal{V}$ : we will see many examples in Section 4 where we can find a set $C$ of simpler identities than those in each basis found for Id $\left(\mathcal{V}^{\prime}\right)$. Of course, by Theorem 2.2, each $e$-variety of regular semigroups is weakly, as well as strongly, determined by each basis of $\operatorname{Id}\left(\mathcal{V}^{\prime}\right)$.

Example 2.3. Of course $(\mathcal{R S})^{\prime}=\mathcal{R U S}$, and a basis for $\operatorname{Id}(\mathcal{R U} \mathcal{S})$ is $(x y) z=x(y z)$, $x x^{\prime} x=x$, and $x^{\prime} x x^{\prime}=x^{\prime}$. Not every subvariety of $R \mathcal{S}$ is of the form $\mathcal{V}^{\prime}$ for some $e$-variety $\mathcal{V}$. In fact $\mathcal{R Z}$, the e-variety of right zero semigroups (those satisfying the identity $x y=y$ ), has only two e-subvarieties (itself and the trivial variety), but the corresponding variety $(\mathcal{R Z})^{\prime}$ has infinitely many subvarieties: if $x^{(0)}=x$ and $x^{(n)}=\left(x^{(n-1)}\right)^{\prime}$, for $n=1,2,3, \ldots$, then $(\mathcal{R Z})^{\prime}$ has a subvariety determined by $x^{(m)}=x^{(m+n)}$ for each pair $(m, n)$ of non-negative integers. For each non-negative integer $\mathrm{k}$, there is also a subvariety determined by $x^{(k)}=y^{(k)}$. These varieties are all the subvarieties of $(\mathcal{R Z})^{\prime}$, which are of course essentially the known varieties of algebras with one operation only, a unary operation.

Example 2.4. The equational class $\mathcal{E}\left((x y)^{\prime}=x^{\prime} y^{\prime}\right)$ contains all right zero senigroups and all left zero semigroups (those satisfying $x y=x$ ), but it does not contain the direct product $S \times U$ of a left zero semigroup $S=\{s, t\}$ and a right zero semigroup $U=\{u, v\}$ : any function ' from $S \times U$ to $S \times U$ is an inverse unary operation, and for many choices of ', $\left(S \times U, \cdot,^{\prime}\right)$ will not satisfy $(x y)^{\prime}=x^{\prime} y^{\prime}$. Hence $\mathcal{E}\left((x y)^{\prime}=x^{\prime} y^{\prime}\right)$ is not closed under $P$, and so is not an e-variety.

\section{The LATTICE OF e-VARIETIES}

Since the map $\mathcal{V} \rightarrow \mathcal{V}^{\prime}$ is one-to-one, from the class $\mathcal{L}_{\text {ev }}(\mathcal{R} S)$ of e-varieties of regular semigroups into the set $\mathcal{L}_{v}(\mathcal{R U S})$ of varieties of regular unary semigroups, the class $\mathcal{L}_{\text {ev }}(\mathcal{R} S)$ is actually a set. Moreover, since the intersection of any set of $e$-varieties is again an e-variety, we have that the set $\mathcal{L}_{\text {ev }}(\mathcal{R S})$ is a (complete) lattice under class containment $\subseteq$, and for any set $\left\{\mathcal{V}_{i}: i \in I\right\}$ of e-varieties, we have

$$
\bigwedge_{i \in I} \mathcal{V}_{i}=\bigcap_{i \in I} \mathcal{V}_{i}
$$


and

$$
\bigvee_{i \in I} \mathcal{V}_{i}=\bigcap\left\{\mathcal{V} \in \mathcal{L}_{\mathrm{ev}}(\mathcal{R} \mathcal{S}):(\forall i \in I)\left(\mathcal{V}_{i} \subseteq \mathcal{V}\right)\right\}
$$

the least $e$-variety containing $\bigcup_{i \in I} \mathcal{V}_{i}$.

Clearly the map ': $\mathcal{L}_{\text {ev }}(\mathcal{R S}) \rightarrow \mathcal{L}_{v}(\mathcal{R U S}), \mathcal{V} \rightarrow \mathcal{V}^{\prime}$, is order-preserving: we will see that it is $\wedge$-preserving but not $\vee$-preserving.

LEMMA 3.1. The map ': $\mathcal{L}_{\text {ev }}(\mathcal{R S}) \rightarrow \mathcal{L}_{v}(\mathcal{R U S}), \mathcal{V} \rightarrow \mathcal{V}^{\prime}$, is $\wedge$-preserving.

Proof: For any set $\left\{\mathcal{V}_{i}: i \in I\right\}$ of $e$-varieties, we have

$$
\begin{aligned}
\left(\bigcap_{i \in I} \mathcal{V}_{i}\right)^{\prime} & =\left\{\left(S, \cdot,^{\prime}\right) \in \mathcal{R U S}:(S, \cdot) \in \bigcap_{i \in I} \mathcal{V}_{i}\right\} \\
& =\left\{\left(S, \cdot,^{\prime}\right) \in \mathcal{R U S}:(\forall i \in I)\left((S, \cdot) \in \mathcal{V}_{i}\right)\right\} \\
& =\bigcap_{i \in I}\left\{\left(S, \cdot,^{\prime}\right) \in \mathcal{R U S}:(S, \cdot) \in \mathcal{V}_{i}\right\} \\
& =\bigcap_{i \in I} \mathcal{V}_{i}^{\prime}, \text { as required. }
\end{aligned}
$$

Remark 3.2. All that we have done so far holds equally for universal algebras. For a fixed type $\mathcal{T}$ of algebra, let

$$
u_{i}\left(x_{1}, \ldots, x_{m}, y_{1}, \ldots, y_{n}\right)=v_{i}\left(x_{1}, \ldots, x_{m}, y_{1}, \ldots, y_{n}\right), \text { for } i \in I,
$$

be a set of identities, and let $\mathcal{E}$ be the class of all algebras $\mathrm{A}=\left\langle A, F^{\mathbf{A}}\right\rangle$ of type $\mathcal{T}$ (where $A$ is the underlying set of $A$ and $F^{A}$ is the list of operations of $A$ ) such that for any elements $a_{1}, \ldots, a_{m} \in A$ there exist elements $b_{1}, \ldots, b_{n} \in A$ such that

$$
u_{i}\left(a_{1}, \ldots, a_{m}, b_{1}, \ldots, b_{n}\right)=v_{i}\left(a_{1}, \ldots, a_{m}, b_{1}, \ldots, b_{n}\right), \quad \text { for } i \in I
$$

By an e-variety of algebras from $\mathcal{E}$ we mean any subclass $\mathcal{V}$ of $\mathcal{E}$ closed under $\mathbf{H}, \mathbf{S}_{\boldsymbol{e}}$ and $\mathbf{P}$, the operations of taking all homomorphic images, subalgebras that are also in $\mathcal{E}$, and products, respectively.

To each algebra $\mathbf{A}$ in $\mathcal{E}$ we can adjoin $m$-ary operations $f_{1}^{\mathbf{A}}, f_{2}^{\mathbf{A}}, \ldots, f_{n}^{\mathbf{A}}$, obtaining $\left\langle A, F^{\mathbf{A}}, f_{1}^{\mathbf{A}}, f_{2}^{\mathbf{A}}, \ldots, f_{n}^{\mathbf{A}}\right\rangle$, as follows: for each $m$-tuple $\left(a_{1}, a_{2}, \ldots, a_{m}\right)$ of elements of $A$, choose $b_{1}, \ldots, b_{n} \in A$ such that equations (1) hold, and define $f_{j}^{A}\left(a_{1}, \ldots, a_{m}\right)=b_{j}$, for $j=1,2, \ldots, n$. For each subclass $\mathcal{C}$ of $\mathcal{E}$ we define $\mathcal{C}^{\prime}$ to consist of algebras $\left\langle A, F^{\mathbf{A}}, f_{1}^{\mathbf{A}}, f_{2}^{\mathbf{A}}, \ldots, f_{n}^{\mathbf{A}}\right\rangle$ such that $\mathrm{A}=\left\langle A, F^{\mathbf{A}}\right\rangle$ belongs to $\mathcal{C}$ and $b_{1}, \ldots, b_{n}$ are chosen in all the ways that make equations (1) valid in $A$. 
Then just as in Theorem 2.1 , for each e-variety $\mathcal{V}$, the class $\mathcal{V}^{\prime}$ is a variety; $\mathcal{V}$ is determined by each basis $B$ of the identities $\operatorname{Id}\left(\mathcal{V}^{\prime}\right)$ as in Theorem 2.2, and the map $\mathcal{V} \rightarrow \mathcal{V}^{\prime}$ is order preserving and $\wedge$-preserving from $\mathcal{L}_{\text {ev }}(\mathcal{E})$, the lattice of $e$-varieties contained in $\mathcal{E}$, to the lattice $\mathcal{L}_{v}\left(\mathcal{E}^{\prime}\right)$ of subvarieties of $\mathcal{E}^{\prime}$, as in Lemma 3.1.

Example 3.3. For $\mathcal{L} \mathcal{Z}$ and $\mathcal{R Z}$, the e-varieties of left zero and right zero semigroups respectively, we have that $\mathcal{L Z} \vee \mathcal{R Z}=\mathcal{R B}$, the $e$-variety of all rectangular bands: we show that $(\mathcal{L Z})^{\prime} \vee(\mathcal{R Z})^{\prime} \neq(\mathcal{R B})^{\prime}$. Now each member of $(\mathcal{L} \mathcal{Z})^{\prime}$ and of $(\mathcal{R Z})^{\prime}$, and hence also each member of $(\mathcal{L Z})^{\prime} \vee(\mathcal{R Z})^{\prime}$, satisfies the identity $(x y)^{\prime}=x^{\prime} y^{\prime}$. But if $(S, \cdot)$ is a rectangular band, then $\left(S, \cdot,^{\prime}\right) \in(\mathcal{R B})^{\prime}$ for any function ' from $S$ to $S$, and as claimed in Example 2.4, it is easy to find $S$ and ' not satisfying $(x y)^{\prime}=x^{\prime} y^{\prime}$.

We define a (forgetful) function $\mathcal{F}: \mathcal{R U S} \rightarrow \mathcal{R S}$ by $\left(S, \cdot{ }^{\prime}\right) \mathcal{F}=(S, \cdot)$, for each regular unary semigroup $\left(S, \cdot,^{\prime}\right)$. For each class $\mathcal{W} \subseteq \mathcal{R U S}$, we define $\mathcal{W} \mathcal{F}=\{(S, \cdot) \in$ $\left.\mathcal{R S}:\left(S, \cdot,^{\prime}\right) \in \mathcal{W}\right\}$.

Example 3.4. Here we find a variety $\mathcal{W} \in \mathcal{L}_{v}(\mathcal{R U S})$ such that $\mathcal{W F}$ is not an e-variety.

Let $S$ be the combinatorial completely 0 -simple semigroup $\mathcal{M}^{0}(\langle 1\rangle ; 3,2 ; P)$ where

$$
P=\left(\begin{array}{lll}
0 & 1 & 1 \\
1 & 0 & 1
\end{array}\right)
$$

Denote by $a$ and $b$ the two non-idempotent elements of $S$, and define ' on $S$ by $a^{\prime}=b$, $b^{\prime}=a$ and $e^{\prime}=e$ for each idempotent $e \in S$.

Then for each $s \in S$, the one-generator unary subsemigroup $\langle s\rangle\left(=\left\langle\left\{s, s^{\prime}\right\}\right\rangle\right)$ generated by $s$ is an inverse unary subsemigroup. It is clear that the class $(\mathcal{I U S})^{1}$ of all regular unary semigroups whose one-generator unary subsemigroups are inverse (unary) semigroups, is a subvariety of $\mathcal{R U S}$.

Now $T=\mathcal{M}^{0}\left(\langle 1\rangle ; 2,2 ;\left(\begin{array}{ll}0 & 1 \\ 1 & 1\end{array}\right)\right)$ is a subsemigroup of $(S, \cdot)$, so $T$ is in the $e$-variety generated by $(S, \cdot)$, while $T \notin(\mathcal{I U S})^{\mathbf{1}} \mathcal{F}$ (also $T \notin\left\langle\left(S, \cdot,^{\prime}\right)\right\rangle_{v} \mathcal{F}$ ), whence $(\mathcal{I U S})^{1} \mathcal{F}$ and $\langle(S, \cdot, ')\rangle_{v} \mathcal{F}$ are not closed under $S_{e}$ and so are not $e$-varieties. (By $\left\langle\left(S, \cdot,{ }^{\prime}\right)\right\rangle_{v}$ we mean the $\mathcal{R U S}$ variety generated by $\left.\left(S, \cdot,{ }^{\prime}\right).\right)$

Example 3.5. This is another example of a variety $\mathcal{W} \in \mathcal{L}_{v}(\mathcal{R U S})$ such that $\mathcal{W} \mathcal{F}$ is not an e-variety: here $\mathcal{W F}$ is not closed under taking morphic images, and not closed under taking regular subsemigroups. Let $\mathcal{W}$ be the variety determimed by the identity $x^{\prime \prime}=x$ (so in particular, the unary operation is one-to-one and onto). The semigroup $S=\mathcal{M}^{0}(\langle 1\rangle ; 4,2 ; P)$, where

$$
P=\left(\begin{array}{llll}
1 & 1 & 0 & 0 \\
0 & 0 & 1 & 1
\end{array}\right)
$$


is in $\mathcal{W F}$, while the semigroup $T=\mathcal{M}^{0}(\langle 1\rangle ; 3,2 ; Q)$, where

$$
Q=\left(\begin{array}{lll}
1 & 0 & 0 \\
0 & 1 & 1
\end{array}\right),
$$

is not in $\mathcal{W F}$, though $T$ is a morphic image (and a regular subsemigroup) of $S$.

We have seen examples of $e$-varieties $\mathcal{U}, \mathcal{V}$ such that $\mathcal{U}^{\prime} \vee \mathcal{V}^{\prime} \neq(\mathcal{U} \vee \mathcal{V})^{\prime}$. A natural question to ask is whether $\left(\mathcal{U}^{\prime} \vee \mathcal{V}^{\prime}\right) \mathcal{F}=\mathcal{U} \vee \mathcal{V}$ for all $e$-varieties $\mathcal{U}$ and $\mathcal{V}$.

Example 3.6. (joint with K.G. Johnston). Here we produce $e$-varieties $\mathcal{U}$ and $\mathcal{V}$ for which $\left(\mathcal{U}^{\prime} \vee \mathcal{V}^{\prime}\right) \mathcal{F} \neq \mathcal{U} \vee \mathcal{V}$. In fact $\left(\mathcal{U}^{\prime} \vee \mathcal{V}^{\prime}\right) \mathcal{F}$ is not an e-variety, though $\mathcal{U}^{\prime} \vee \mathcal{V}^{\prime}$ is a more complicated $\mathcal{R} U \mathcal{S}$ variety than those in Examples 3.4 and 3.5.

Let $\mathcal{U}$ be the $e$-variety generated by the combinatorial Brandt semigroups: then $\mathcal{U}^{\prime}=\left\langle\left(B_{2}, \cdot,^{-1}\right)\right\rangle_{v}$, the inverse semigroup variety of $[12$, Section XII.4] (therein denoted by $\mathcal{B}$ ), which is generated by the five element combinatorial Brandt semigroup $\left(B_{2}, \cdot,^{-1}\right)$. Put $\mathcal{V}=\mathcal{R Z}$, the $e$-variety of right zero semigroups. Both $\mathcal{U}^{\prime}$ and $\mathcal{V}^{\prime}$ (and also the variety of all inverse semigroups) satisfy the identities $(x y)^{\prime \prime}=x^{\prime \prime} y^{\prime \prime}$ and $(x y x)^{\prime}=x^{\prime} y^{\prime} x^{\prime}$, so $\mathcal{U}^{\prime} \vee \mathcal{V}^{\prime}$ satisfies these identities also.

The semigroup $M=\mathcal{M}^{0}(\langle f\rangle ; 2,3 ; P)$, where

$$
P=\left(\begin{array}{ll}
f & 0 \\
0 & f \\
0 & f
\end{array}\right)
$$

(and where $\langle f\rangle$ denotes a trivial group with element $f$ ), is easily seen to be a morphic image of $B_{2} \times(R Z)_{2}$, where $(R Z)_{2}$ is a two element right zero semigroup (so $M \in \mathcal{U V}$ $\mathcal{V})$. Put $T=\mathcal{M}^{0}(\langle e\rangle ; 3,3 ; \triangle)$, the ten element combinatorial Brandt semigroup (where $\langle e\rangle$ is also a one element group), put $T^{*}=T \backslash\{0\}$, and define a partial homomorphism [2, Section 3.2] $\phi: T^{*} \rightarrow M$ as follows: $(e ; i, j) \rightarrow(f ; i, j),(e ; 3, j) \rightarrow(f ; 2, j)$, for $i=1,2$ and $j=1,2,3$. Let $S=T^{*} \cup M$ be the semigroup [2, Theorem 4.19] which is the ideal extension of $M$ by $T$ determined by $\phi$.

It is easy to see that $S$ is isomorphic to the subsemigroup $\{(t, t \phi) \in T \times M: t \in$ $\left.T^{*}\right\} \cup(\{0\} \times M)$ of $T \times M$ (this is a special case of [11, Theorem 4.1]), and so $S \in \mathcal{U} \vee \mathcal{V}$. Finally we show that $S \notin\left(\mathcal{U}^{\prime} \vee \mathcal{V}^{\prime}\right) \mathcal{F}$ by showing there is no inverse unary operation ' on $S$ satisfying the identity $(x y x)^{\prime}=x^{\prime} y^{\prime} x^{\prime}$. Suppose, to the contrary, that there is such an operation ' on $S$. Then in $\left(S, \cdot,^{\prime}\right)$ we have

$$
\begin{aligned}
& (e ; 3,1)(f ; 2,1)^{\prime}(e ; 3,1) \\
& =(e ; 3,1) \phi(f ; 2,1)^{\prime}(e ; 3,1) \phi \\
& =(f ; 2,1)(f ; 2,1)^{\prime}(f ; 2,1)=(f ; 2,1) .
\end{aligned}
$$


Thus

$$
\begin{aligned}
(f ; 2,1)^{\prime} & =\left[(e ; 3,1)(f ; 2,1)^{\prime}(e ; 3,1)\right]^{\prime} \\
& =(e ; 3,1)^{\prime}(f ; 2,1)^{\prime \prime}(e ; 3,1)^{\prime} \\
& =(e ; 1,3)(f ; 2,1)(e ; 1,3)
\end{aligned}
$$

(by uniqueness of inverses of

$$
(e ; 3,1),(f ; 1,2) \text { and }(f ; 1,3))
$$$$
=(e ; 1,3) \phi(f ; 2,1)(e ; 1,3) \phi
$$$$
=(f ; 1,3)(f ; 2,1)(f ; 1,3)
$$$$
=(f ; 1,3) \text {. }
$$

Similarly we can prove that $(f ; 2,1)^{\prime}=(f ; 1,2)$, whence $(f ; 1,2)=(f ; 1,3)$, a contradiction. Thus $S \notin\left(\mathcal{U}^{\prime} \vee \mathcal{V}^{\prime}\right) \mathcal{F}$, so $\left(\mathcal{U}^{\prime} \vee \mathcal{V}^{\prime}\right) \mathcal{F}$ is not an e-variety, and in particular $\left(\mathcal{U}^{\prime} \vee \mathcal{V}^{\prime}\right) \mathcal{F} \neq \mathcal{U} \vee \mathcal{V}$. We remark that

(1) a similar proof shows that for every inverse unary operation on $(S, \cdot)$, $\left(S, \cdot,^{\prime}\right)$ does not satisfy $(x y)^{\prime \prime}=x^{\prime \prime} y^{\prime \prime}$; and

(2) a similar example can be obtained by replacing $T$ in the construction by the 0 -direct union of two copies of $B_{2}=\mathcal{M}^{0}(\langle 1\rangle ; 2,2 ; \Delta)$.

\section{IDENTITIES FOR e-VARIETIES}

Here we give identities [strongly] determining many of the known existence varieties of regular semigroups.

\subsection{Completely REgUlar SEMigroups}

By a completely regular (or $\mathcal{C R}$ ) semigroup, $(S, \cdot)$, we mean a semigroup which is a union of groups, while by a completely regular unary $\left(\right.$ or $\left.(\mathcal{C R})^{-1}\right)$ semigroup $\left(S, \cdot,^{-1}\right)$ we mean a completely regular senigroup $(S, \cdot)$ together with the unary operation ${ }^{-1}$ which assigns to each element $x$ its inverse $x^{-1}$ in the maximal subgroup containing $x$. It is well-known that any regular subsemigroup of a $\mathcal{C R}$ semigroup is also a $\mathcal{C R}$ semigroup (a corollary to [6, Result 2(iii)]; and also to Lemma 4.1.1 below). It follows that $\mathcal{C R}$ is an e-variety, and that each e-subvariety of $\mathcal{C} \mathcal{R}$ consists of $\mathcal{C} \mathcal{R}$ semigroups.

Just as for the $e$-variety $\mathcal{C R}$ of all completely regular semigroups, each $\epsilon$-subvariety $\mathcal{V}$ of $\mathcal{C R}$ can be altered to a variety of $(\mathcal{C R})^{-1}$ semigroups by simply adjoining to each semigroup $S$ in $\mathcal{V}$ the unary operation ${ }^{-1}$; we denote this variety by $\mathcal{V}^{-1}$.

Conversely, each variety $\mathcal{W}$ of completely regular unary semigroups becomes an $e$-variety $\mathcal{W} \mathcal{F}$ by simply dropping the unary operation ${ }^{-1}$ from each semigroup. Meets are the same, and joins are the same, in $\mathcal{L}_{e v}(\mathcal{C R})$, the lattice of $e$-varieties of completely 
regular semigroups, as in $\mathcal{L}_{v}\left[(\mathcal{C R})^{-1}\right]$, the lattice of varieties of completely regular unary semigroups. Thus we see that $\mathcal{L}_{\text {ev }}(\mathcal{C R}) \cong \mathcal{L}_{v}\left[(\mathcal{C R})^{-1}\right]$.

However, the identities determining a $(\mathcal{C R})^{-1}$ variety $\mathcal{W}$ are not always appropriate to determine the $\mathcal{C R}$-variety $\mathcal{W F}$ : for example the identity $x x^{-1}=x^{-1} x$, true in any $(\mathcal{C R})^{-1}$ semigroup, when translated to $x x^{\prime}=x^{\prime} x$, weakly determines the $e$-variety of all semilattices of groups (since now, by $S$ satisfies $x x^{\prime}=x^{\prime} x$, we mean that $x x^{\prime}=x^{\prime} x$ for every inverse $x^{\prime}$ of $x$ in $S$ ).

We find an identity that determines $\mathcal{C R}$ and we show how to translate identities for $(\mathcal{C R})^{-1}$ varieties to ideutities for $\mathcal{C R}$ e-varieties.

LEMMA 4.1.1. For any element $x$ in any $(\mathcal{C R})^{-1} \operatorname{semigroup}\left(S, \cdot,{ }^{-1}\right)$,

$$
x^{-1}=x^{m}\left[x^{m+n+1}\right]^{\prime} x^{n}
$$

for any positive integers $m, n$ and for any inverse $\left[x^{m+n+1}\right]^{\prime}$ of $x^{m+n+1}$.

Proof: We have

$$
\begin{aligned}
x^{m}\left[x^{m+n+1}\right]^{\prime} x^{n} & =x^{-n-1} x^{m+n+1}\left[x^{m+n+1}\right]^{\prime} x^{m+n+1} x^{-m-1} \\
& =x^{-n-1} x^{m+n+1} x^{-m-1}=x^{-1} .
\end{aligned}
$$

ThEOREM 4.1.2. The e-variety $\mathcal{C R}$ is strongly determined within $\mathcal{R S}$ by the identity

$$
x x^{-1} x=x,
$$

where $x^{-1}=x\left(x^{3}\right)^{\prime} x$.

Proof: From Lemma 4.1.1, any $\mathcal{C R}$ semigroup satisfies $x x^{-1} x=x$. Conversely, take any regular semigroup $(S, \cdot)$ such that for some inverse unary operation ' on $(S, \cdot)$, $\left(S, \cdot,^{\prime}\right)$ satisfies $x x^{-1} x=x$, that is, satisfies $x=x^{2}\left(x^{3}\right)^{\prime} x^{2}$. Then, for all $x \in S$, $H_{x} \leqslant H_{x^{2}} \leqslant H_{x}$ (that is, $R_{x} \leqslant R_{x^{2}} \leqslant R_{x}$ and $L_{x} \leqslant L_{x^{2}} \leqslant L_{x}$ ), whence $H_{x^{2}}=H_{x}$ and $H_{x}$ is a group, so $(S, \cdot)$ is a union of groups. This proves the theorem.

For any $(\mathcal{C R})^{-1}$ semigroup word $u=u\left(x_{1}, x_{2}, \ldots, x_{n}\right)$ we define the translation $u^{T}$ of $u$ inductively, as follows: for each variable $x, x^{T}=x$ and $\left(x^{-1}\right)^{T}=x\left(x^{3}\right)^{\prime} x$; for each word $w,\left(w^{-1}\right)^{T}=w^{T}\left[\left(w^{T}\right)^{3}\right]^{\prime} w^{T}$; for any words $v, w,(v w)^{T}=v^{T} w^{T}$. An identity $u=v$ for a $(\mathcal{C R})^{-1}$ variety then translates to $u^{T}=v^{T}$ for a $\mathcal{C R} e$-variety. It is clear that if a set of identities $\left(u_{i}=v_{i}\right)_{i \in I}$ determines a $(\mathcal{C R})^{-1}$ variety $\mathcal{W}$ then $\left(u_{i}^{T}=v_{i}^{T}\right)_{i \in I}$, together with $x x^{-1} x=x$, strongly determine the $e$-variety $\mathcal{W F}$. Since 
$u^{T}$ will often be much longer than $u$, in practice one would not translate $\left(u_{i}=v_{i}\right)_{i \in I}$ to $\left(u_{i}^{T}=v_{i}^{T}\right)_{i \in I}$ but would retain the identities $\left(u_{i}=v_{i}\right)_{i \in I}$, regarding each $w^{-1}$ as meaning $w^{T}\left[\left(w^{T}\right)^{3}\right]^{\prime} w^{T}$. One would thus still regard $\left(u_{i}=v_{i}\right)_{i \in I}$ as strongly determining $\mathcal{W} \mathcal{F}$ within the e-variety $\mathcal{C} \mathcal{R}$.

We note that each variety $\mathcal{V}$ of bands is determined (within the variety of all bands) by some single (semigroup) identity $u=v$, as in $\left[1,3\right.$ or 5]. Clearly $x^{2}=x$, $u=v$ determine $\mathcal{V}$ as an $e$-variety of regular semigroups (and as a variety of $(\mathcal{C R})^{-1}$ semigroups, once the unary operation ${ }^{-1}$ has been adjoined).

\subsection{INVERSE SEMIGROUPS}

Since a unary semigroup $\left(S,,^{-1}\right)$ is an inverse semigroup [12, Theorem XII.1.1] if and only it satisfies the identities $x(y z)=(x y) z, x x^{-1} x=x,\left(x^{-1}\right)^{-1}=x,(x y)^{-1}=$ $y^{-1} x^{-1},\left(x x^{-1}\right)\left(x^{-1} x\right)=\left(x^{-1} x\right)\left(x x^{-1}\right)$, we see that the existence variety $\mathcal{I}$ of inverse semigroups is strongly determined within $\mathcal{R S}$ by $x^{\prime \prime}=x,(x y)^{\prime}=y^{\prime} x^{\prime}$ and $\left(x x^{\prime}\right)\left(x^{\prime} x\right)=$ $\left(x^{\prime} x\right)\left(x x^{\prime}\right)$. Note that by Remark 4.3 .4 below, $\mathcal{I}$ is strongly determined within $\mathcal{R} S$ by the single identity

$$
\left[x\left(x^{2}\right)^{\prime} x\right]\left[y\left(y^{2}\right)^{\prime} y\right]=\left[y\left(y^{2}\right)^{\prime} y\right]\left[x\left(x^{2}\right)^{\prime} x\right]
$$

If we merely regard $x^{-1}$ as an alternative notation to $x^{\prime}$ when dealing with $e$ varieties of inverse semigroups, then any $e$-variety $\mathcal{V}$ of inverse semigroups is strongly determined within $\mathcal{I}$ by each set of identities that determines $\mathcal{V}^{\prime}$ withing $\mathcal{I}^{\prime}$, and of course $\mathcal{V}^{\prime}$ is an inverse semigroup variety in the earlier sense (see, for example, [12, Chapter XII]). Clearly $\mathcal{L}_{\mathrm{ev}}(\mathcal{I})$, the lattice of e-varieties of inverse semigroups, is isomorphic to $\mathcal{L}_{v}\left(I^{\prime}\right)$, the lattice of varieties of inverse unary semigroups.

LEMMA 4.2.1.

(a) The identity $(x y)^{\prime}=y^{\prime} x^{\prime}$ weakly determines $\mathcal{I}$ within $\mathcal{R S}$.

(b) The identity $x x^{\prime} x^{\prime} x=x^{\prime} x x x^{\prime}$ weakly determines $\mathcal{I}$ within $\mathcal{R} S$.

Proof: (a) Take any regular semigroup $(S, \cdot)$ such that $\left(S, \cdot,^{\prime}\right)$ satisfies $(x y)^{\prime}=$ $y^{\prime} x^{\prime}$ for every inverse unary operation ' on $(S, \cdot)$. Take any $\mathcal{R}$-related idempotents $e, f$ in $S$ and define $e^{\prime}=e, f^{\prime}=f$, and choose $x^{\prime} \in V(x)$ arbitrarily for $x \in S \backslash\{e, f\}$. Then $(e f)^{\prime}=f^{\prime} e^{\prime}$ gives $f=e$, so each $\mathcal{R}$-class, and similarly each $\mathcal{L}$-class, of $(S, \cdot)$ contains exactly one idempotent, whence $(S, \cdot)$ is an inverse semigroup [9, Theorem V.1.2]. Conversely if $(S, \cdot)$ is an inverse semigroup, then $\left(S, \cdot,^{\prime}\right)$ satisfies $(x y)^{\prime}=y^{\prime} x^{\prime}$ for any (in fact, the unique) inverse unary operation' on $(S, \cdot)$.

(b) Take any regular semigroup $(S, \cdot)$ such that $\left(S, \cdot,,^{\prime}\right)$ satisfies $x x^{\prime} x^{\prime} x=x^{\prime} x x x^{\prime}$ for every inverse unary operation ' on $(S, \cdot)$. Take any $\mathcal{R}$-related idempotents $e, f$ in $S$, and define $e^{\prime}=f, f^{\prime}=e$, and choose $x^{\prime} \in V(x)$ arbitrarily for $x \in S \backslash\{e, f\}$. 
Then $e e^{\prime} e^{\prime} e=e^{\prime} e e e^{\prime}$ becomes $e=f$. Again, each $\mathcal{R}$-class, and similarly each $\mathcal{L}$-class, of $S$ contains exactly one idempotent, so $(S, \cdot)$ is an inverse semigroup. The converse implication is again trivial.

Remark 4.2.2. The lattices $\mathcal{L}_{v}\left(I^{\prime}\right)$ and $\mathcal{L}_{v}\left((\mathcal{C R})^{-1}\right)$ (of varieties of inverse and completely regular semigroups, respectively) are of course also sublattices of $\mathcal{L}_{v}(\mathcal{R} U S)$. In [13], Petrich and Reilly determined the joins in $\mathcal{L}_{v}(\mathcal{R U S})$ of the varieties of rectangular bands and the varieties of strict inverse semigroups (that is, subdirect products of Brandt semigroups and groups). In $\mathcal{L}_{\text {ev }}(\mathcal{R S})$, the corresponding (quite different) joins can be simply described, as we shall see in a further paper. For example $\mathcal{R B} \vee \mathcal{I}$, the join of the $e$-varieties of rectangular bands and inverse semigroups is the e-variety of generalised inverse semigroups, and more generally, the join $\mathcal{R B} \vee \mathcal{V}$, for each e-variety $\mathcal{V}$ of inverse semigroups, is just the class of generalised inverse semigroups $S$ such that $S / \gamma \in \mathcal{V}$, where $\gamma$ is the least inverse semigroup congruence on $S$.

Remark 4.2.3. In [7], the author, jointly with T. Imaoka, showed that the $e$-variety of generalised inverse semigroups has the amalgamation property. In the further paper mentioned in Remark 4.2.2, we will show that this is the largest e-variety in $\mathcal{L}_{\text {ev }}(\mathcal{R} S)$ with the amalgamation property.

\subsection{REgULAR SEMIGROUPS WITH CORES IN AN $e$-VARIETY $\mathcal{V}$}

By the core of a semigroup $S$, we mean the subsemigroup Core $(S)=\langle E(S)\rangle$ generated by the set $E=E(S)$ of all idempotents of $S$.

For each class $\mathcal{C}$ of regular semigroups define

$$
\mathcal{C}^{i g}=\{S \in \mathcal{R S}: \text { Core }(S) \in \mathcal{C}\},
$$

the class of those regular senigroups whose core is in $\mathcal{C}$.

Lemma 4.3.1. For each existence variety $\mathcal{V}$, the class $\mathcal{V}^{i g}$ is also an existence variety.

Proof: For any set $\left\{S_{i}: i \in I\right\}$ of semigroups, we have Core $\left[\prod_{i \in I} S_{i}\right] \subseteq \prod_{i \in I} \operatorname{Core}\left(S_{i}\right)$. (For $I$ finite, equality holds, and for $I$ infinite, examples show that equality does not always hold.) For any semigroup $S$ and any subsemigroup $T$, we have Core $(T) \subseteq$ Core $(S)$. And for any regular semigroups $S$ and $T$ and any morphism $\phi: S \rightarrow T$ of $S$ onto $T$, we have, from Lallement's Lemma $[9$, Lemma 11.4.6], that Core $(T)=$ Core $(S) \phi$. The lemma now follows.

Remark 4.3.2. By [6, Theorem 3], a regular semigroup $S$ is $E$-solid if and only if Core $(S)$ is a union of groups. Thus the class of all $E$-solid regular semigroups is precisely $\mathcal{C} \mathcal{R}^{i g}$, and is an e-variety.

Given a set of identities for $\mathcal{V}$, a set for $\mathcal{V}^{i g}$ can be obtained as follows. 
THEOREM 4.3.3. Let $\left[u_{i}\left(x_{1}, \ldots, x_{k_{i}}\right)=v_{i}\left(x_{1}, \ldots, x_{k_{i}}\right)\right]_{i \in I}$ be a set of RUS identities that weakly [strongly] determine an $e$-variety $\mathcal{V}$. Then $\mathcal{V}^{i g}$ is weakly [strongly] determined within $\mathcal{R S}$ by the identities

$$
\begin{gathered}
u_{i}\left[w_{1, n(1)}, \ldots, w_{k_{i}, n\left(k_{i}\right)}\right]=v_{i}\left[w_{1, n(1)}, \ldots, w_{k_{i}, n\left(k_{i}\right)}\right], \\
\text { for all } i \in I, \text { for all } n(1), \ldots, n\left(k_{i}\right) \in \mathcal{Z}^{+},
\end{gathered}
$$

where, for $j=1,2, \ldots$ (or for $j=1,2, \ldots, \max _{i \in I} k_{i}$ if $\max _{i \in I} k_{i}$ exists),

$$
w_{j, n(j)}=\left[y_{j, 1}\left[\left(y_{j, 1}\right)^{2}\right]^{\prime} y_{j, 1}\right] \ldots\left[y_{j, n(j)}\left[\left(y_{j, n(j)}\right)^{2}\right]^{\prime} y_{j, n(j)}\right]
$$

(a product of $n(j)$ idempotents).

Proof: The theorem can be proved routinely from the observations, for any $\left(S, \cdot,^{\prime}\right) \in \mathcal{R} U S$, that (i) $x\left(x^{2}\right)^{\prime} x$ is an idempotent, for any $x \in S$, (ii) if $e$ is any idempotent in $S$, then $e=e\left(e^{2}\right)^{\prime} e$, and (iii) Core $(S)$ is closed under ', by FitzGerald's Lemma ([4], or [8, Exercise II.15]).

Remark 4.3.4. In particular, since $\mathcal{I}=(\mathcal{S L})^{i g}$, where $\mathcal{S L}$ is the $e$-variety of semilattices, we have that the $e$-variety $\mathcal{I}$ of inverse semigroups is strongly determined within $\mathcal{R S}$ by the identity

$$
\left[x\left(x^{2}\right)^{\prime} x\right]\left[y\left(y^{2}\right)^{\prime} y\right]=\left[y\left(y^{2}\right)^{\prime} y\right]\left[x\left(x^{2}\right)^{\prime} x\right]
$$

since $\mathcal{S L}$ is strongly determined by $x^{2}=x, x y=y x$. Hence the variety of inverse (unary) semigroups is determined by the identities

$$
\begin{gathered}
(x y) z=x(y z), x x^{-1} x=x, x^{-1} x x^{-1}=x^{-1} \\
\left(x\left(x^{2}\right)^{-1} x\right)\left(y\left(y^{2}\right)^{-1} y\right)=\left(y\left(y^{2}\right)^{-1} y\right)\left(x\left(x^{2}\right)^{-1} x\right) .
\end{gathered}
$$

\subsection{ORThODOX SEMIGROUPS}

For any $\mathcal{R} U S$ word $u$, by $u \in E$ we mean the identity $u^{2}=u$ (as in [12] for inverse semigroups).

THEorem 4.4.1. The e-variety $\mathcal{O}$ of all orthodox semigroups is strongly determined within $R S$ by the three identities

$$
\left(x x^{\prime}\right)\left(x^{\prime} x\right),\left(x^{\prime} x\right)\left(x x^{\prime}\right),\left(x x^{\prime} x^{\prime} x\right)\left(y y^{\prime} y^{\prime} y\right) \in E ;
$$

and also by the identity

$$
x\left(x^{2}\right)^{\prime} x y\left(y^{2}\right)^{\prime} y \in E
$$


And $\mathcal{O}$ is weakly determined within $\mathcal{R S}$ by each one of the following four identities:

$$
x x^{\prime} y y^{\prime} \in E, x x^{\prime} x^{\prime} x \in E,\left(x\left(x^{2}\right)^{\prime} x\right)^{\prime} \in E, x y y^{\prime} x^{\prime} x y=x y .
$$

Proof: (i) Take any orthodox senigroup $(S, \cdot)$ and any regular unary operation ' on $(S, \cdot)$. Clearly $\left(S, \cdot,,^{\prime}\right)$ satisfies the identities $(3)$ and $\left(3^{\prime}\right)$ (since $x\left(x^{2}\right)^{\prime} x$ is an idempotent, for each $x \in S$ ), and the first two identities in (4). From [9, Theorem VI.1.1], we see that $\left(S, \cdot,^{\prime}\right)$ satisfies the last two identities in (4).

(ii) Conversely, take any regular semigroup $(S, \cdot)$ such that for some inverse unary operation ' on $(S, \cdot)$, the identities $(3)$ are satisfied by $\left(S, \cdot,^{\prime}\right)$. We show that $(S, \cdot)$ is orthodox. Take any idempotents $e, f \in S$. By (3), $e^{\prime}=e^{\prime} e e^{\prime}=\left(e^{\prime} e\right)\left(e e^{\prime}\right) \in E$ and hence $e=e e^{\prime} e=\left(e e^{\prime}\right)\left(e^{\prime} e\right)$. Likewise $f=\left(f f^{\prime}\right)\left(f^{\prime} f\right)$ and then from (3) again $e f$ is idempotent, whence $(S, \cdot)$ is orthodox.

For $\left(3^{\prime}\right)$, there is a similar, though simpler, proof (since $e=e\left(e^{2}\right)^{\prime} e$ for any idempotent $e \in S$ ).

(iii) Take any regular semigroup $(S, \cdot)$.

If $(S, \cdot)$ satisfies the first of the identities (4), then clearly $(S, \cdot)$ is orthodox.

Suppose $(S, \cdot)$ satisfies $x x^{\prime} x^{\prime} x \in E$, and take any two idempotents $e, f \in S$. By [6, Lemma 1], there exist idempotents $g, h$ such that $g h=$ ef $\mathcal{D} g \mathcal{D} h$. Since $g \mathcal{D} h$ there exist $x \in S$ and $y \in V(x)$ such that $x y=g$ and $y x=h$. Define $x^{\prime}=y$ and choose $s^{\prime} \in V(s)$ arbitrarily for $s \in S \backslash\{x\}$. Then ef $=g h=x x^{\prime} x^{\prime} x \in E$, so $(S, \cdot)$ is orthodox. (The result here is equivalent to the result that a regular semigroup is orthodox if and only if the product of each pair of $\mathcal{D}$-related idempotents is also idempotent, which follows from [6, Lenuma 1].)

Suppose $(S, \cdot)$ satisfies $\left(x\left(x^{2}\right)^{\prime} x\right)^{\prime} \in E$ and take any idempotent $e \in S$ and any $y \in V(e)$. Define $e^{\prime}=y$ and choose $s^{\prime} \in V(s)$ arbitrarily for $s \in S \backslash\{e\}$. Then $y=e^{\prime}=\left(e e^{\prime} e\right)^{\prime}=\left(e\left(e^{2}\right)^{\prime} e\right)^{\prime} \in E$, so by [0, Theorem VI.1.1], $(S, \cdot)$ is orthodox.

Suppose $(S, \cdot)$ satisfies $x y y^{\prime} x^{\prime} x y=x y$, and take any idempotents $e, f \in S$. Define $e^{\prime}=e, f^{\prime}=f$, and choose $s^{\prime} \in V(s)$ arbitrarily for $s \in S \backslash\{e, f\}$. Then $(e f)^{2}=e f e f=e\left(f f^{\prime}\right)\left(e^{\prime} e\right) f=(e f)\left(f^{\prime} e^{\prime}\right)(e f)=e f$, so $e f$ is idempotent and $(S, \cdot)$ is orthodox.

Remark 4.4.2. For any $\mathcal{R} U S$ words $u$ and $v$, by $u \in V(v)$ we mean the pair of identities $u v u=u$ and $v u v=v$. From the theorem, $\mathcal{O}$ is (weakly) determined by $y^{\prime} x^{\prime} \in V(x y)$.

Recall again that each (semigroup) variety of bands is determined by a single semigroup identity $([1,3$ and 5$])$.

Theorem 4.4.3. If a band variety $\mathcal{V}$ is determined by a semigroup identity 
$u\left(x_{1}, x_{2}, \ldots, x_{n}\right)=v\left(x_{1}, x_{2}, \ldots, x_{n}\right)$ then the existence variety $\mathcal{V}^{i g}$, of all orthodox semigroups $S$ such that $E(S) \in \mathcal{V}$, is strongly determined within $\mathcal{R} S$ by the identities (3) or (3') together with

$$
u\left(x_{1} x_{1}^{\prime} x_{1}^{\prime} x_{1}, \ldots, x_{n} x_{n}^{\prime} x_{n}^{\prime} x_{n}\right)=v\left(x_{1} x_{1}^{\prime} x_{1}^{\prime} x_{1}, \ldots, x_{n} x_{n}^{\prime} x_{n}^{\prime} x_{n}\right)
$$

and also by (3) or (3') together with

$$
u\left(x_{1}\left(x_{1}^{2}\right)^{\prime} x_{1}, \ldots, x_{n}\left(x_{n}^{2}\right)^{\prime} x_{n}\right)=v\left(x_{1}\left(x_{1}^{2}\right)^{\prime} x_{1}, \ldots, x_{n}\left(x_{n}^{2}\right)^{\prime} x_{n}\right) .
$$

Also $\mathcal{V}^{i g}$ is weakly determined by each one of the identities (4) and the identity

$$
u\left(x_{1} x_{1}^{\prime}, x_{2} x_{2}^{\prime}, \ldots, x_{n} x_{n}^{\prime}\right)=v\left(x_{1} x_{1}^{\prime}, x_{2} x_{2}^{\prime}, \ldots, x_{n} x_{n}^{\prime}\right)
$$

Proof: For any idempotents $x_{1}, x_{2}, \ldots, x_{n}$ in an orthodox semigroup $(S, \cdot)$, we have

$$
x_{i}=x_{i} x_{i}^{\prime} x_{i}^{\prime} x_{i} \text { and } x_{i}=x_{i}\left(x_{i}^{2}\right)^{\prime} x_{i}, i=1,2, \ldots, n \text {, }
$$

for any inverse unary operation ' on $(S, \cdot)$. The theorem follows easily from this and Theorem 4.4.1.

\subsection{E-SOLID REGULAR SEMIGROUPS}

For any elements $a, b$ in a regular semigroup $S, R_{a} \geqslant R_{b}$ if and only if $a a^{*} b=b$ for each [some] $a^{*} \in V(a)$. Let us write $a \geqslant_{\mathcal{R}} b$ (and $b \leqslant_{\mathcal{R}} a$ ) to mean $R_{a} \geqslant R_{b}$ and likewise $a \geqslant_{\mathcal{L}} b$ (and $b \leqslant_{\mathcal{L}} a$ ) to mean $L_{a} \geqslant L_{b}$.

For any two $\mathcal{R U S}$ words $u, v$, the notation $u \leqslant_{\mathcal{R}} v$ (or $v \geqslant_{\mathcal{R}} u$ ) means the identity $v v^{\prime} u=u$. By $u \mathcal{R} v$ we mean the conjunction of $u \leqslant_{\mathcal{R}} v$ and $u \geqslant_{\mathcal{R}} v$. Of course $u \leqslant_{\mathcal{C}} v, u \geqslant_{\mathcal{C}} v$ and $u \mathcal{L} v$ are defiued dually, while $u \mathcal{H} v$ means the conjunction of $u \mathcal{L} v$ and $u \mathcal{R} v$. Finally, $u \in G$ means $u \mathcal{H} u^{2}$, namely that $u$ is in a subgroup (as in [12] for inverse semigroups).

We recall that the class of all $E$-solid regular semigroups is the existence variety $\mathcal{H} \mathcal{R}^{i g}$ (Remark 4.3.2).

ThEOREM 4.5.1. The existence variety $\mathcal{C} \mathcal{R}^{i g}$ of all $E$-solid regular semigroups is strongly determined within $R S$ by the twelve identities

$$
\left(x^{\prime} x\right)\left(x x^{\prime}\right),\left(x x^{\prime}\right)\left(x^{\prime} x\right),\left(x x^{\prime} x^{\prime} x\right)\left(y y^{\prime} y^{\prime} y\right) \in G
$$

and also by the four identities

$$
x\left(x^{2}\right)^{\prime} x y\left(y^{2}\right)^{\prime} y \in G
$$


And $\mathcal{C R}^{\text {ig }}$ is weakly determined within $\mathcal{R} S$ by each of the following sets of four identities:

$$
\begin{aligned}
& x x^{\prime} y y^{\prime} \in G ; \\
& x x^{\prime} x^{\prime} x \in G ;
\end{aligned}
$$

and

$$
\left(x\left(x^{2}\right)^{\prime} x\right)^{\prime} \in G
$$

Proof: (i) Let $(S, \cdot)$ be any $E$-solid regular semigroup and let ' be any inverse unary operation on $(S, \cdot)$. Since the core $\langle E(S)\rangle$ of $S$ is a union of groups, the identities (5) and $\left(5^{\prime}\right)$ are satisfied by $\left(S, \cdot,^{\prime}\right)$.

(ii) Conversely, suppose $(S, \cdot)$ is a regular semigroup such that $\left(S, \cdot,^{\prime}\right)$ satisfies the identities (5), for some inverse unary operation ' on $(S, \cdot)$. Take any idempotents $e, f, g$ in $S$ such that $e \mathcal{L} f \mathcal{R} g$. Then $e^{\prime}=e^{\prime} e e^{\prime}=\left(e^{\prime} e\right)\left(e e^{\prime}\right)$ is in a subgroup, by (5), and since $e \mathcal{R} e e^{\prime} \mathcal{L} e^{\prime} \mathcal{R} e^{\prime} e \mathcal{L} e$ we have by [2, Theorem 2.17] that $\left(e e^{\prime}\right)\left(e^{\prime} e\right) \in H_{e}$ (since $R_{e^{\prime} e} \cap L_{e e^{\prime}}=H_{e^{\prime}}$ (a group) contains an idempotent).

Likewise $\left(g g^{\prime}\right)\left(g^{\prime} g\right) \in H_{g}$, and since $e \mathcal{L} f \mathcal{R} g$ we have by [2, Theorem 2.17] again that $\left(e e^{\prime} e^{\prime} e\right)\left(g g^{\prime} g^{\prime} g\right) \in R_{e} \cap L_{g}$, and so $R_{e} \cap L_{g}$ is a group from (5); that is, there is an idempotent $h$ such that e $\mathcal{R} h \mathcal{L} g$, whence $S$ is $E$-solid, as required.

Now take any regular semigroup $(S, \cdot)$ such that $\left(S, \cdot,^{\prime}\right)$ satisfies $\left(5^{\prime}\right)$ for some inverse unary operation ' on $(S, \cdot)$. Then clearly the product of any two idempotents in $(S, \cdot)$ is in a subgroup, which is equivalent to $(S, \cdot)$ being $E$-solid.

(iii) The identities $(6)\left[\left(6^{\prime}\right)\right]$ are equivalent to the product of any two [D-related] idempotents being in a subgroup, which is equivalent to being $E$-solid. The identities (7) are equivalent to each inverse of an idempotent being in a subgroup, which is also equivalent to being $E$-solid.

\subsection{LOCALLY INVERSE, ORTHODOX,...}

Let $\mathcal{V}$ be any class of regular semigroups and define $\mathcal{V}^{\text {loc }}$ to be the class of all regular semigroups all of whose local subsemigroups are in $\mathcal{V}$; that is

$$
\mathcal{V}^{l o c}=\{(S, \cdot) \in \mathcal{R} S:(e S e, \cdot) \in \mathcal{V} \text { for each idempotent } e \in S\}
$$

The operalor $\mathcal{V} \rightarrow \mathcal{V}^{\text {loc }}$ was introduced for $(\mathcal{C R})^{-1}$ varieties by the author and P.R. Jones $[8$, Section 4$]$, and was denoted by $P$. 
LEMMA 4.6.1. If $\mathcal{V}$ is an existence variety then $\mathcal{V}^{\text {loc }}$ is also an existence variety.

Proof: It is clear that $\mathcal{V}^{l o c}$ is closed under taking regular subsemigroups and direct products.

Take any regular semigroups $S, T$ and any morphism $\phi: S \rightarrow T$ of $S$ onto $T$. Take any idempotent $f \in T$. By Lallement's Lemma [9, Lenma II.4.6] there is an idempotent $e \in S$ such that $e \phi=f$. It is easily checked that $(e S e) \phi=f T f$, so each local subsemigroup of $T$ is a morphic image of a local subsemigroup of $S$. Thus $\mathcal{V}^{\text {loc }}$ is closed under morphic images, and hence is an $e$-variety.

We call $\mathcal{V}^{\text {loc }}$ the existence variety of regular locally- $\mathcal{V}$ semigroups. As in [8, Proposition 4.1] for $(\mathcal{C R})^{-1}$ varieties, the operator $\mathcal{V} \rightarrow \mathcal{V}^{\text {loc }}$ is a closure operator on the lattice $\mathcal{L}_{\text {ev }}(\mathcal{R} S)$, and in particular on the lattice of [existence] varieties of inverse semigroups.

We now show how to obtain identities for $\mathcal{V}^{\text {loc }}$ from identities for $\mathcal{V}$. For any idempotent $e$ in any regular semigroup $S$, and for any element $x \in e S e$, and for any inverse $x^{\prime}$ of $x$ in $S$, checking shows that $e x^{\prime} e$ is an inverse of $x$ in $e S e$ (in particular $e S e$ is a regular subsenugroup of $S$ ). Also if $e$ and $f$ are $\mathcal{D}$-related idempotents in any semigroup $S$, then, as is well-known, $e S e$ is isomorphic to $f S f$ (and so $e S e \in \mathcal{V}$ would imply $f S f \in \mathcal{V}$ ).

For any $R U S$ word $w=w\left(x_{1}, x_{2}, \ldots, x_{n}\right)$ in variables $x_{1}, x_{2}, \ldots, x_{n}$, we define a word $w^{\text {loc }}$ in variables $x_{1}, x_{2}, \ldots, x_{n}, y$, inductively, as follows: $x_{i}^{\text {loc }}=y y^{\prime} x_{i} y y^{\prime}$, $i=1,2, \ldots, n ;\left(u^{\prime}\right)^{l o c}=y y^{\prime}\left(u^{l o c}\right)^{\prime} y y^{\prime}$; and $(u v)^{l o c}=u^{l o c} v^{l o c}$ (for any words $u, v$ in $\left.x_{1}, \ldots, x_{n}\right)$.

Remark 4.6.2. A slight simplification of the definition of $w^{\text {loc }}$ occurs by defining $x_{i}^{l o c}=y x_{i} y^{\prime}$ (instead of $\left.y y^{\prime} x_{i} y y^{\prime}\right)$ for each variable $x_{i}$. The theorem below is still valid, with $y x_{i} y^{\prime}$ replacing $y y^{\prime} x_{i} y y^{\prime}$.

THEOREM 4.6.3. If a set of $\mathcal{R U S}$ identities

$$
u_{i}\left(x_{1}, x_{2}, \ldots, x_{k_{i}}\right)=v_{i}\left(x_{1}, x_{2}, \ldots, x_{k_{i}}\right), \text { for } i \in I
$$

weakly [strongly] determines an e-variety $\mathcal{V}$ of regular semigroups, then $\mathcal{V}^{\text {loc }}$ is weakly [strongly] determined by the identities

$\left((8)^{l o c}\right) \quad u_{i}^{l o c}\left(x_{1}, x_{2}, \ldots, x_{k_{i}}, y\right)=v_{i}^{l o c}\left(x_{1}, x_{2}, \ldots, x_{k_{i}}, y\right)$, for $i \in I$

Proof: Case (a): the identities (8) weakly detemine $\mathcal{V}$.

(i) Take any regular semigroup $(S, \cdot)$ that satisfies the identities $(8)^{\text {loc }}$; we show that $(S, \cdot) \in \mathcal{V}^{l o c}$. 
Take any idempotent $e \in S$ and take any inverse unary operation ' on $\left(e S e,{ }^{\cdot}\right)$, and extend ' arbitrarily to an inverse unary operation, also denoted by ', on $(S, \cdot)$. In $e S e$ we have $V(e)=e$, and so $e^{\prime}=e$. Take any $x_{1}, x_{2}, \ldots, x_{n} \in e S e$, and put $y=e$, whence $y^{\prime}=e=y y^{\prime}$. Now ' is closed on $e S e=y y^{\prime} S y y^{\prime}$, and $y y^{\prime} u y y^{\prime}=u$ for any $u \in e S e$, so

$$
w^{l o c}\left(x_{1}, x_{2}, \ldots, x_{n}, y\right)=w\left(x_{1}, x_{2}, \ldots, x_{n}\right)
$$

for any word $w$ in $n$ variables. Thus, since $\left(S, \cdot,^{\prime}\right)$ satisfies $(8)^{l o c}$, we see that $\left(e S e, \cdot,^{\prime}\right)$ satisfies (8). Since' was arbitrary on $e S e$, we have that $(e S e, \cdot)$ satisfies (8), and so $(e S e, \cdot) \in \mathcal{V}$, giving us that $(S, \cdot) \in \mathcal{V}^{\text {loc }}$.

(ii) Take any senigroup $(S, \cdot) \in \mathcal{V}^{\text {loc }}$. We show that $(S, \cdot)$ satisfies $(8)^{\text {loc }}$. So we take any inverse unary operation ' on $(S, \cdot)$, any $i \in I$, and any elements $x_{1}, x_{2}, \ldots, x_{k_{i}}, y \in S$.

Define a unary operation * on $\left(y y^{\prime} S y y^{\prime}, \cdot\right)$ by $x^{*}=y y^{\prime} x^{\prime} y y^{\prime}$ for each $x \in y y^{\prime} S y y^{\prime}$; then ${ }^{*}$ is an inverse unary operation.

The elements $y y^{\prime} x_{1} y y^{\prime}, \ldots, y y^{\prime} x_{k_{i}} y y^{\prime}$ are in $y y^{\prime} S y y^{\prime}$, and $\left(y y^{\prime} S y y^{\prime}, \cdot\right) \in \mathcal{V}$, whence $\left(y y^{\prime} S y y^{\prime}, .,^{*}\right) \in \mathcal{V}^{\prime}$, so

$$
u_{i}\left(y y^{\prime} x_{1} y y^{\prime}, \ldots, y y^{\prime} x_{k_{i}} y y^{\prime}\right)=v_{i}\left(y y^{\prime} x_{1} y y^{\prime}, \ldots, y y^{\prime} x_{k_{i}} y y^{\prime}\right),
$$

where each side of the equation is calculated in the regular unary semigroup $\left(y y^{\prime}\right.$ Syy', ., *). Hence

$$
u_{i}^{l o c}\left(x_{1}, \ldots, x_{k_{i}}, y\right)=v_{i}^{l o c}\left(x_{1}, \ldots, x_{k_{i}}, y\right) .
$$

Thus $\left(S, \cdot,^{\prime}\right)$ satisfies $(8)^{\text {loc }}$, and so $(S, \cdot)$ satisfies $(8)^{l o c}$.

Case (b): the identities (8) strongly determine $\mathcal{V}$.

(i) Take any regular semigroup $(S, \cdot)$ such that for some inverse unary operation ' on $(S, \cdot)$, the regular unary semigroup $\left(S, \cdot{ }^{\prime}\right)$ satisfies $(8)^{\text {loc }}$ : we show that $(S, \cdot) \in$ $\mathcal{V}^{\text {loc }}$.

Take any idempotent $y \in S$. Since $y \mathcal{R} y y^{\prime}$, we have that $(y S y, \cdot) \cong\left(y y^{\prime} S y y^{\prime}, \cdot\right)$ (the map $x \rightarrow x y y^{\prime}$ is an isomorphism). Again define an inverse unary operation * on $\left(y y^{\prime} S y y^{\prime}, \cdot\right)$ by $x^{*}=y y^{\prime} x^{\prime} y y^{\prime}$ (for all $x \in y y^{\prime} S y y^{\prime}$ ). Since $\left(S, \cdot,^{\prime}\right)$ satisfies the identities $(8)^{\text {loc }}$, we see that $\left(y y^{\prime} S y y^{\prime}, \cdot,{ }^{*}\right)$ satisfies the identities (8) (with * replacing '), that is, belongs to $\mathcal{V}^{\prime}$. Hence $\left(y y^{\prime} S y y^{\prime}, \cdot\right) \in \mathcal{V}$ and so $(y S y, \cdot) \in \mathcal{V}$ (since $(y S y, \cdot) \cong$ $\left.\left(y y^{\prime} S y y^{\prime}, \cdot\right)\right)$, which gives us that $(S, \cdot) \in \mathcal{V}^{\text {loc }}$.

(ii) We showed already in part (a)(ii) of the proof that any semigroup $(S, \cdot) \in$ $\mathcal{V}^{\text {loc }}$ satisfies the identities (8) ${ }^{\text {loc }}$ if each semigroup in $\mathcal{V}$ satisfies (8). The proof is complete. 
As for inverse semigroups [12, Definition II.4.7], let us call a regular semigroup strict if it is a subdirect product of completely $O$-simple and completely simple semigroups. From Lallement [11, Theorem 4.1], a regular semigroup $S$ is strict if and only if for any elements $a, b \in S$ with $J_{a} \geqslant J_{b}$, for each idempotent $e \in J_{a}$ there exists a unique idempotent $f \in J_{b}$ such that $e \geqslant f$. It is now easy to see also that a regular semigroup $S$ is strict if and only if it is locally a semilattice of groups (that is, for each idempotent $e \in S$, the local subsemigroup $e S e$ is a semilattice of groups). Likewise, combinatorial, strict, regular semigroups are easily seen to be precisely regular local semilattices (that is, regular semigroups such that each local subsemigroup is a semilattice).

For any $\mathcal{R} U S$ words $u, v$, the notation $u C W v$ means the identity $u v=v u$ (that is, that $u$ commutes with $v$ ).

Corollary 4.6.4. (i) The e-variety $(\mathcal{S L})^{\text {loc }}$ of regular local semilatices (that is, combinatorial strict regular semigroups) is strongly determined within $\mathcal{R} S$ by the identities

$$
z x z^{\prime} \in E, z x z^{\prime} C W z y z^{\prime} .
$$

(ii) The e-variety $(\mathcal{S C G})^{\text {loc }}$ of regular semigroups that locally are semilattices of groups (that is, strict regular semigroups) is weakly determined within $\mathcal{R S}$ (and is strongly determined within $\mathcal{I}^{\text {loc }}$ ) by the identity

$$
y x y^{\prime} C W y y^{\prime}\left(y x y^{\prime}\right)^{\prime} y y^{\prime} .
$$

(iii) The e-variety $\mathcal{B}^{\text {loc }}$ of regular local bands is strongly determined within $\mathcal{R} S$ by the identity $y x y^{\prime} \in E$.

(iv) The e-variety $\mathcal{O}^{\text {loc }}$ of regular, locally orthodox semigroups is strongly determined within $\mathcal{R S}$ by

$$
\left(z x z^{\prime}\left(\left(z x z^{\prime}\right)^{2}\right)^{\prime} z x z^{\prime}\right)\left(z y z^{\prime}\left(\left(z y z^{\prime}\right)^{2}\right)^{\prime} z y z^{\prime}\right) \in E
$$

and is weakly determined within $\mathcal{R S}$ by

$$
\left(y x\left(y^{\prime}\left(y x y^{\prime}\right)^{\prime} y\right)^{2} x y^{\prime}\right)^{\prime} \in E
$$

(v) The e-variety $\mathcal{I}^{\text {loc }}$ of regular, locally inverse semigroups is strongly determined within $\mathcal{R} S$ by the identity

$$
z x z^{\prime}\left(\left(z x z^{\prime}\right)^{2}\right)^{\prime} z x z^{\prime} C W z y z^{\prime}\left(\left(z y z^{\prime}\right)^{2}\right)^{\prime} z y z^{\prime}
$$


and is weakly determined within $\mathcal{R} S$ by the identity

$$
y y^{\prime}\left(y x y^{\prime}\right) y x y^{\prime} C W y x y^{\prime}\left(y x y^{\prime}\right)^{\prime} y y^{\prime}
$$

Proof: (iv) The second term is a simplification of the term $\left(y x y^{\prime}\left(y y^{\prime}\left(y x y^{\prime}\right)^{\prime} y y^{\prime}\right)^{2} y x y^{\prime}\right)^{\prime}$, which is an inverse of an arbitrary idempotent in $y y^{\prime} S y y^{\prime}$ if $x, y$ are taken as arbitrary elements in an $\mathcal{R U S}$ semigroup $\left(S, \cdot,{ }^{\prime}\right)$.

(v) The first identity is obtained from Remark 4.3.4. The second identity is obtained from $x^{\prime} x C W x x^{\prime}$, which weakly determines $\mathcal{I}$ within $\mathcal{R S}$.

\section{REFERENCES}

[1] A.P. Birjukov, 'Varieties of idempotent semigroups', Algebra i Logika $\theta$ (1970), 255-273. (Russian) .

[2] A.H. Clifford and G.B. Preston, The Algebraic Theory of Semigroups Vol. I (Math. Surveys of the American Math. Soc. 7, Providence, R.I., 1961).

[3] C.F. Fennemore, 'All varieties of bands I and II', Math. Nachr 48 (1971), 237-252 and 253-262.

[4] D.G. FitzGerald, 'On inverses of products of idempotents in regular semigroups', J. Austral. Math. Soc. 13 (1972), 335-337.

[5] J.A. Gerhard, 'The lattice of equational classes of idempotent semigroups', J. Algebra 15 (1970), 195-224.

[6] T.E. Hall, 'On regular semigroups', J. Algebra 24 (1973), 1-24.

[7] T.E. Hall, 'Amalgamation for inverse and generalized inverse semigroups', Trans. Amer. Math. Soc. (to appear).

[8] T.E. Hall and P.R. Jones, 'On the lattice of varieties of bands of groups', Pacific J. Math. 91 (1980), 327-337.

[8] J.M. Howie, An Introduction to Semigroup Theory, London Math. Soc. Monograplss 7 (Academic Press, London, New York, 1976).

[10] Jiłi Kadourek and Maria B. Szendrei, 'A new approach in the theory of orthodox semigroups', (preprint).

[11] G. Lallement, 'Structure theorems for regular semigroups', Semigroup Forum 4 (1972), 95-123.

[12] Mario Petrich, Inverse Semigroups (John Wiley and Sons, 1984).

[13] Mario Petrich and Norman R. Reilly, "The juin of the varieties of strict inverse semigroups and rectangular bands', Glasgow Math. J. 25 (1984), 59-74.

[14] N.R. Reilly and H.E. Scheiblich, 'Congruences on regular semigroups', Pacific J. Math. 23 (1967), 349-360.

Mathematics Department

Monash Uxiversity

Clayton, Victoria 3168

Australia 